\title{
Event
}

\section{Region Bergstraße profitiert von Deutscher Radsportmeisterschaft}

Große Herausforderungen, starker Wettbewerb und die Fähigkeit, kurzfristig Höchstleistungen abzurufen - dadurch zeichneten sich die Leistungen der Radsportler bei der diesjährigen Deutschen Radsportmeisterschaft im Juni aus. Die 26 Kilometer lange Rundstrecke des Straßenradrennens mit einem 7 Kilometer langen Anstieg zum Col de Kuralpe verlangte ihnen alles ab. Am Ende triumphierte Emanuel Buchmann bei den Herren. Zuvor gewannen Trixi Worrack bei den Damen und Lennard Kämna bei den U23-Wettkämpfen. „Leistungsfähigkeit, Teamgeist und Einsatzbereitschaft müssen stimmen. Diese besonderen Herausforderungen kennen wir nicht nur aus dem Leistungssport, sondern auch aus unserem Arbeitsalltag“, sagte Rainer Berthan, Executive Vice President und Geschäftsführer von Sirona bei der Siegerehrung.
Auch die Organisatoren der Veranstaltung haben erstaunliches geleistet. Innerhalb von 3 Monaten hatten die Verantwortlichen in Bensheim und im heimischen Radsportverein SSG Bensheim die Vorbereitungen gestemmt, nachdem Dresden als Austragungsort geplatzt war. Sirona war kurzfristig Hauptsponsor eingesprungen. Berthan zeigte sich sehr zufrieden mit dem SportEvent: „Als Hauptsponsor sind wir natürlich froh, dass alles wunderbar geklappt hat und dass die Veranstal- tung bundesweit so eine große Aufmerksamkeit in den Medien gefunden hat. Die ganze Region Bergstraße profitiert davon.“ Der Dentalgerätehersteller Sirona, größter Arbeitgeber der Region, ist durch seinen Standort Bensheim sehr mit der Bergstraße verbunden und unterstützt seit Jahren regionale Sportmannschaften wie die SSG Bensheim und das Zweitligateam der Damenhandballer „Flames“.

Nach einer Pressemitteilung der Sirona Dental GmbH, A - Wals bei Salzburg

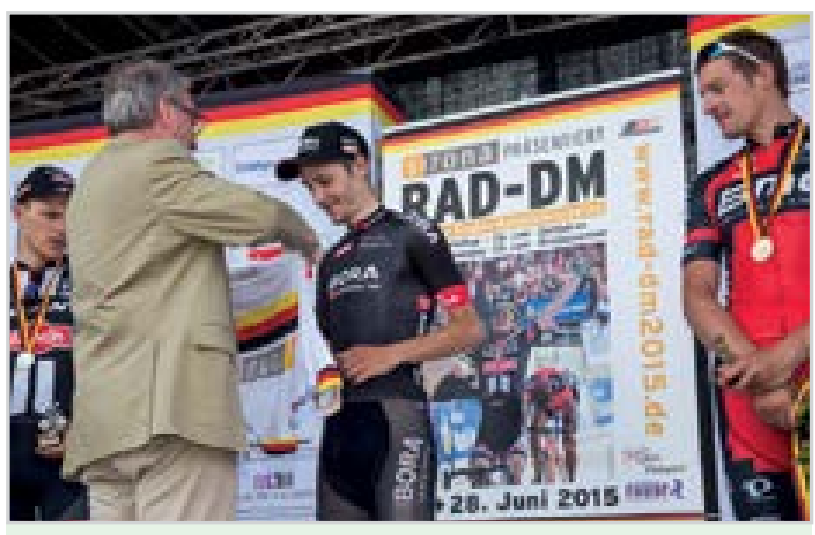

R. Berthan überreichte das Sieger-Trikot an den neuen deutschen Meister E. Buchmann. 